\title{
Does Carbapenem Resistant Enterobacteriaceae Infection Drive Venous Thromboembolism in Patients Admitted to Intensive Care Units Receiving Prophylactic Anticoagulants?
}

Fahad Aleidan ( $\square$ faleidan@gmail.com )

King Saud bin Abdulaziz University for Health Sciences https://orcid.org/0000-0002-4293-3947

Sara Albilal

King Abdulaziz Medical City

Maha Alammari

King Abdulaziz Medical City

Khalid Al Sulaiman

King Abdulaziz Medical City

Mohammed Alassiri

King Saud bin Abdulaziz University for Health Sciences

Abdel Galil Abdel Gadir

King Saud bin Abdulaziz University for Health Sciences

\section{Research}

Keywords: Carbapenem-resistant Enterobactereaceae, infection, intensive care, venous thromboembolism, prophylactic anticoagulants

Posted Date: January 14th, 2021

DOl: https://doi.org/10.21203/rs.3.rs-143671/v1

License: (c) (i) This work is licensed under a Creative Commons Attribution 4.0 International License. Read Full License 


\section{Abstract}

\section{Background}

Systemic infections are one of several risk factors leading to the development of inflammation and venous thromboembolism (VTE) formation.

Aim of the study

To assess the risk factors associated with the development of VTE during the stay of critically ill patients in the intensive care unit (ICU).

Materials and methods

This is a matched case-control study of patients with VTE admitted to the ICU, at a single centre, from January 12018 to December 31 2019. We included all adult patients who stayed more than two days before the development of VTE. Conditional logistic regression was used to estimate the odds ratio (OR) for the risk factors for VTE.

Results

Univariate and multivariate analyses uncovered three of six factors to have significant influence in the development of VTE in ICU patients: Carbapenem-resistant Enterobactereaceae (CRE) infections (OR 2.95, $95 \%$ confidence interval $(\mathrm{Cl}) 1.21-7.33, p=.010)$, length of ICU stay (OR 1.02, 95\% $\mathrm{Cl} 1.01-1.04, p=.011)$ and the sequential organ failure assessment (SOFA) score (OR 1.10, 95\% $\mathrm{Cl} 1.01-1.20, p=.031$ ); all were found to be independent risk factors in the development of VTE.

Conclusion

Our findings suggest that in patients admitted to the ICU, CRE infection is a strong trigger to the development of VTE, and draw the attention of the treating clinicians to prioritise these infections in the management protocols to control infection-driven VTE in ICU patients.

\section{Introduction}

Venous thromboembolism (VTE), also known as deep vein thrombosis, (DVT) is a condition in which a blood clot forms most often in the deep veins of the leg, groin or arm and travels in the venous circulation, lodging in the lungs (known as pulmonary embolism, PE). It is a serious condition that is usually associated with high rates of morbidity, mortality and cost [1]. Three prothrombotic factors are recognized to contribute to underlie the development of VTE, the Virchow's triad: venous stasis, endothelial damage, and a hypercoagulable state [2]. VTE, in general, has long been considered a disease that affects predominantly European populations, a misconception resulting from the paucity of 
epidemiological data from non-European countries [3]. It is now evident that VTE is prevalent across a number of different racial groups [4].

This issue has become of interest in view of the observation that ethnicity and or race need to be considered not only when resorting to thromboprophylaxis but also when assessing the known risk factors for VTE [5]. In special patient groups, such as critically-ill patients, the risk of VTE is increased substantially particularly in those patients who are admitted to the intensive care units (ICUs) [6, 7]. Although ICU patients share similar general risk factors for VTE with other hospital patients: immobilization, age, obesity, sepsis, pregnancy, past personal or familial history of VTE, they have additional risk factors associated with ICU admission including among others, infection, mechanical ventilation, (by decreasing venous return) and prolonged stay in the ICU $[8,9]$. Acute systemic or localized infections increase the risk of thrombosis up-to 20 times and are independent risk factors for VTE [10, 11]. Furthermore, the risk of infection associated with thrombosis is highest when the infection is active, or in the weeks shortly afterwards. In addition, immobilisation brings with it venous stasis, one of the three basic prothrombotic mechanisms of the Virchow's triad $[12,13]$. In this respect prophylactic doses of anticoagulants, whether unfractionated heparin (UFH) or low-molecular-weight-heparin (LMWH) were repeatedly shown to lower the risk of hospital-related life threatening VTEs [14-16]. Again, the available data on the effectiveness of thromboprophylaxis in critically-ill patients has emerged largely from European populations $[17,18]$. In view of the scarcity of information on both VTE as well as thrombophylaxis in Middle Eastern populations, further research is certainly needed to characterize all aspects of VTE particularly the risk factors associated with it. The current study was undertaken to identify the risk factors of VTE among ICU patients receiving prophylactic anticoagulants.

\section{Materials And Methods}

This retrospective single-centre observational study was performed between January 1st 2018 and December 31st 2019. All the patients who were included in the study were admitted to the ICUs of the King Abdulaziz Medical City (KAMC), Riyadh, Saudi Arabia and the patients' data were obtained from the BestCare ${ }^{\circledR}$ database, KAMC system. The collected data included the following demographic and clinical parameters: Age, sex, body mass index (BMI), comorbid diseases (history of cancer, hypertension, diabetes mellites, pulmonary diseases, cardiovascular diseases, liver diseases and chronic kidney diseases, as well as the severity of the disease classification which was calculated using the Acute Physiology and Chronic Health Evaluation II (APACHE II) score, ranging from 0 to 71[19]. The sequential Organ Failure Assessment (SOFA) score, which represents the severity of the disorder in any of the 6 vital organs of the body systems: respiratory, coagulation, cardiovascular and circulatory, liver, central nervous system and renal, and was scored on a 0-4 scale [20]. Additionally, the Nutrition Risk In The Critically ill (NUTRIC) score was used to identify patients who will benefit from aggressive nutrition [21]. The following baseline laboratory data were obtained: platelet count, serum creatinine and haemoglobin. Additionally, the following outcome data were also recorded: ICU length of stay (LOS), number of patients using mechanical ventilation, bleeding events, infection acquisition during the ICU stay and death. The patient inclusion criteria were: age equal to or greater than 18 years; the ICU stay for more than 2 days, 
patient on VTE prophylaxis (guideline-recommended thrombophylaxis) using either subcutaneous low molecular weight heparin or unfractionated heparin. The exclusion criteria were: patients admitted to ICU due to VTE or VTE diagnosed within 24 hours of ICU admission; patients receiving therapeutic doses of heparin or on oral anticoagulants during ICU stay; patients who died or discharged within 48 hours after ICU admission. All patients who fulfilled our inclusion criteria during the study period were considered VTE study cases. The controls were patients without VTE events who were randomly selected in a ratio of 1:1 patient to controls and were matched for age ( \pm 2 years), sex, and the same ICU and period of admission. The Institutional Review Board (IRB) approval was obtained from the King Abdullah International Medical Research Centre (KAIMRC) and informed consent was waived.

\section{Statistical analysis}

The analysis of data was performed using the Statistical Package for the Social Sciences (SPSS) software (USA, version 26). All $p$-values were two sided and a value $<.05$ was considered statistically significant. The Pearson's chi square test was used to compare the categorical variables. Normally distributed continuous variables were analysed using the independent $t$-test and the Mann-Whitney $U$ test was used to compare median of non-normal distribution continuous variables and the diseases severity scores (APACHE II, SOFA and NUTRIC). Binary logistic regression was also used in the analysis. The univariate and multivariate analyses were employed to identify the risk factors for the development of VTE.

\section{Results}

234 patients were identified with VTE admitted to the ICUs at KAMC in the period between January 1st 2018 and December 31st 2019. 77 patients were excluded as they met one or more exclusion criteria and 157 patients were included in the final analysis (Fig. 1). The Baseline characteristics of these patients are presented in Table 1. Overall, $51 \%$ of the patients are women and their mean for all patients was $60.8 \pm$ 17 years (range from 24 to 88 years) and the average BMI was $33.9 \pm 5.2 \mathrm{~kg} / \mathrm{m}^{2}$. There was no statistical difference between VTE cases and control group in age, sex, BMI, comorbidities, use of antiplatelets or the anticoagulant prophylaxis received. 
Table 1

Demographic characteristics of patients

\begin{tabular}{|c|c|c|c|}
\hline Variables & $\begin{array}{l}\text { VTE } \\
n=157\end{array}$ & $\begin{array}{l}\text { Control } \\
n=157\end{array}$ & $P$ value \\
\hline Age, year $( \pm S D)$ & $61.9( \pm 16)$ & $60.1( \pm 19)$ & .355 \\
\hline Sex, $(\%)$ & $84(53.5)$ & $77(49.0)$ & .249 \\
\hline Female & $73(46.5)$ & $80(51.0)$ & \\
\hline \multicolumn{4}{|l|}{ Male } \\
\hline Obesity, BMI > 30 (\%) & $66(42.0)$ & $58(36.9)$ & .210 \\
\hline \multicolumn{4}{|l|}{ Comorbidities } \\
\hline Diabetes mellitus (\%) & $98(62.4)$ & $89(56.7)$ & .301 \\
\hline Hypertension (\%) & $92(58.6)$ & $80(51.0)$ & .174 \\
\hline Hyperlipidaemia (\%) & $40(25.5)$ & $30(19.1)$ & .175 \\
\hline Cardiovascular diseases (\%) & $22(14.0)$ & $17(10.8)$ & .392 \\
\hline Pulmonary diseases (\%) & $18(11.5)$ & $21(13.4)$ & .608 \\
\hline Chronic kidney diseases (\%) & $26(16.6)$ & $21(13.4)$ & .429 \\
\hline Liver diseases (\%) & $14(8.9)$ & $17(10.8)$ & .570 \\
\hline Oncology diseases (\%) & $28(17.8)$ & $19(12.1)$ & .155 \\
\hline Prophylactic anticoagulants (\%) & & & .734 \\
\hline Unfractionated heparin & $70(44.6)$ & $73(46.5)$ & \\
\hline Low-molecular-weight-heparin & $87(55.4)$ & $84(53.5)$ & \\
\hline Antiplatelets (\%) & $41(26.1)$ & $47(29.9)$ & .450 \\
\hline \multicolumn{4}{|l|}{ Laboratory } \\
\hline Albumin, mean $( \pm S D)$ & $26.4 \pm 8.3$ & $27.9 \pm 8.1$ & .095 \\
\hline Serum creatinine, mean $( \pm S D)$ & $79.7 \pm 58$ & $73.1 \pm 46$ & .254 \\
\hline WBC, mean $( \pm S D)$ & $14.1 \pm 6.8$ & $12.9 \pm 7.3$ & .050 \\
\hline Platelet count, mean ( \pm SD) & $222 \pm 133$ & $211 \pm 138$ & .456 \\
\hline Hemoglobin, mean $( \pm S D)$ & $110 \pm 24$ & $112 \pm 27$ & .355 \\
\hline
\end{tabular}


There were significant differences between VTE patients and the control group in the APACHE II, SOFA and NUTRIC scores; the medians and interquartile ranges were 22 (16-26) vs 18 (12-24, $p=.001), 9$ (6$11)$ vs $7(4-9, p<.0001)$ and $5(4-7)$ vs $4(3-6, p=.004)$, respectively (Table 2$)$. Significantly larger number of VTE patients were on mechanical ventilation compared to controls $(79.9 \%$ vs $64.3 \%, p=.003)$. Patients with VTE stayed longer days in ICU than patients in the control group ( $25.1 \pm 23.8$ vs $17.1 \pm 11.7$ days, $p<.0001)$. Significantly higher proportion of VTE patients acquired CRE infections during their stay in the ICU and before the development of VTE $(21.7 \%$ vs $9.6 \%, p=.003)$. A total of 16 patients $(5.7 \%$ vs $4.4 \%, p=.608$ ) had minor bleeding, but no major bleeding episodes were recorded. The mortality rate was higher in VTE patients compared to control group, $25.5 \%$ vs $8.3 \%, p<.0001$ (Table 2).

Table 2

Assessment of disease severity, VTE risk factors and mortality

\begin{tabular}{|c|c|c|c|}
\hline \multirow[t]{2}{*}{ Variables } & VTE & Control & \multirow[t]{2}{*}{$P$ value } \\
\hline & $n=157$ & $n=157$ & \\
\hline \multicolumn{4}{|l|}{ Assessment of disease severity } \\
\hline APACHE II score, median (IQR) & $22(16-26)$ & $18(12-24)$ & .001 \\
\hline SOFA score, median(IQR) & $9(6-11)$ & $7(4-9)$ & $<.0001$ \\
\hline NUTRIC score, median (IQR) & $5(4-7)$ & $4(3-6)$ & .004 \\
\hline \multicolumn{4}{|l|}{ Risk factors } \\
\hline Mechanical ventilation (\%) & $125(79.6)$ & $101(64.3)$ & .003 \\
\hline CRE infection (\%) & $34(21.7)$ & $15(9.6)$ & .003 \\
\hline ICU length of stay, days ( \pm SD) & $25.1( \pm 23.8)$ & $17.1( \pm 11.7)$ & $<.0001$ \\
\hline Mortality (\%) & $40(25.5)$ & $13(8.3)$ & $<.0001$ \\
\hline \multicolumn{4}{|c|}{$\begin{array}{l}\text { APACHE II, acute physiology and chronic health evaluation II; CRE, carbapenem resistant } \\
\text { Enterobacteriaceae; IQR, interquartile range; ICU, intensive care; NUTRIC, nutrition risk in the critically } \\
\text { ill; SOFA, sequential Organ Failure Assessment; SD, standard deviation; VTE, venous } \\
\text { thromboembolism. }\end{array}$} \\
\hline
\end{tabular}

The results of the univariate and multivariate analyses are presented in Table 3. Three of six risk factors were found to have a significant influence in the development of VTE in ICU patients. CRE infections [odds ratio (OR) 2.95, 95\% confidence interval (Cl) 1.21-7.33, $p=.010$ ], ICU length of stay (OR 1.02, 95\% $\mathrm{Cl} 1.01-1.04, p=.011)$ and SOFA score (OR 1.10, 95\% Cl 1.01-1.20, $p=.031)$ were independent risk factors in the development of VTE. 
Table 3

Univariate and multivariate logistic regression analysis of the risk factors for VTE

\section{Univariate}

Multivariate

\begin{tabular}{|lllllll|}
\hline Variables & Odd ratio & $95 \% \mathrm{Cl}$ & $P$ value & Odd ratio & $95 \% \mathrm{Cl}$ & $P$ value \\
CRE infection & 3.16 & $1.94-6.83$ & .003 & 2.95 & $1.21-7.33$ & .010 \\
\hline ICU length of stay & 1.03 & $1.01-1.04$ & .001 & 1.02 & $1.01-1.04$ & .011 \\
\hline Mechanical Ventilation & 2.17 & $1.30-3.60$ & .003 & 1.58 & $0.91-2.77$ & .106 \\
\hline APACHE II score & 1.04 & $1.01-1.07$ & .006 & 1.01 & $0.98-1.10$ & .506 \\
\hline SOFA score & 1.14 & $1.07-1.22$ & $<.0001$ & 1.10 & $1.01-1.20$ & .031 \\
NUTRIC score & 1.17 & $1.05-1.31$ & .004 & 1.02 & $0.87-1.19$ & .834 \\
$\begin{array}{l}\text { APACHE II, acute physiology and chronic health evaluation II; CRE, carbapenem resistant } \\
\text { Enterobacteriaceae; IQR, interquartile range; ICU, intensive care; NUTRIC, nutrition risk in the critically } \\
\text { ill; SOFA, sequential organ failure assessment; VTE, venous thromboembolism. }\end{array}$ \\
\hline
\end{tabular}

\section{Discussion}

Over the last few decades, evidence has been mounting that VTE is as prevalent in non-Europeans as in Europeans; however, little is known about VTE in former populations [22]; this includes the risk factors associated with the development of VTE.

The present study is a retrospective study carried out in ICU patients with VTE, receiving guidelineanticoagulant thromboprophylaxis; VTE is known to be a very frequent complication in critically ill patients and quite often with negative impact on patients' outcome [10, 18, 23-25]. These earlier studies agree that the use of anticoagulant thromboprophylaxis is associated with a significant reduction in the risk of VTE in these critically-ill patients. In the current study we aimed to identify the risk factors for VTE among ICU Saudi patients receiving prophylactic anticoagulants. It is already well established that ICU patients, other than the general risk factors associated with the development of VTE, such as age, obesity, sepsis, immobilization, malignancy, history of thrombotic disease, have their own risk factors, some of which may compound the general risk factors for VTE and these include, sepsis, immobilization (resulting from sedation), mechanical ventilation, cardio-respiratory failure and end stage renal failure $[8,9,26-28]$.

There are wide disagreements among previous reports on the independent risk factors for VTE. The univariate and multivariate analysis of our data have shown that infection, longer stay in the ICU and mechanical ventilation were the most significant independent risk factors for VTE. Zhang et al [6] found that the use central venous catheter $(P=.002, O R=4.50)$, Caprini score $(P=.012, O R=1.20)$, and ICU length of stay $(P=.006, O R=1.08)$ were independent risk factors related to the development of VTE in patients admitted to the ICU. On the other hand, Kaplan et al [29] in their study of ICU patients with severe sepsis and septic shock identified the increased length of ICU stay as the only risk factor significantly 
associated with development of VTE. Shorr and Williams [30] in their earlier larger randomized controlled trial in critically ill patients with sepsis found that history of VTE was the only risk factor independently associated with the development of VTE (odds ratio, 3.66, 95\% confidence interval 1.77-7.56, $p=0.005$ ). Hong et al [31] in their survey among critically ill patients with DVT of the lower extremity identified age, gender and BMI as significant risk factors for deep vein thrombosis development $(p<.05)$; ten of their patients $(11 \%)$ developed deep vein thrombosis during their stay in the intensive care units.

These wide disagreements on the independent risk factors significantly associated with the development of VTE is not un-expected as different studies vary widely in the inclusion criteria of their critically ill patients, their co-morbidities, the clinical setting and last not least the ethnicity of their patients. Considerations should also take into account the disparity in the study design particularly sample size, whether the study is prospective or retrospective. It is no wonder; therefore, there are wide disagreements in the independent risk factors significantly associated with the development of VTE. These disagreements make it difficult to make meaningful comparisons between the findings of different published reports.

Nonetheless infection and/or sepsis seem to be the most frequent independent risk factor associated with, the development of VTE in these published reports. In the current study, CRE infection was found to be the most frequent trigger for the development of VTE and after unconditional multivariate adjustment, it has also resulted in an increased the risk of VTE by almost 3-fold after unconditional multivariate adjustment. Similarly, in the American Health and Retirement nationwide Study, aiming to identify the most common trigger to the development of VTE in hospitalized elderly patients $(n=399)$ infection was also shown to be the most common reason for hospitalization for venous thromboembolism and has also increased the risk for the development of VTE by 2.9-fold [32].

The important question that needs to be addressed in this respect is about the link between infection and the development of thrombosis. Recently emerging information on the pathophysiological mechanisms of infection-driven thrombosis and VTE has come from in the recent account of Iba et al, [9] who have proposed that microbial products trigger the production and release of inflammatory mediators called pathogen-associated molecular patterns (PAMPs) and damage-associated molecular patterns (DAMPs) during sepsis. Both products activate the coagulation system and trigger the formation of a thrombus. It was also suggested that the increased risk of $\mathrm{PE}$, in particular, might be related to local inflammation in the infected area of the lung. This is compounded by the hypoxic lung environment leading to local vasoconstriction and activation of coagulation [33]. There is also the possibility that severe infection and the associated inflammatory process including the release of pro-inflammatory cytokines would result in activation of the coagulation system and localized thrombotic complications [34].

The other independent risk factor that was significantly associated with the development of VTE was the SOFA score, which was originally developed to give objective quantisation of the degree of the severity of the disease and organ failure of vital body systems including coagulation in patients with sepsis [20]. In patients with sepsis-induced disseminated intravascular coagulation (DIC), Iba et al [35] found that SOFA 
is a useful score in predicting outcomes including mortality. Additionally, The SOFA score has the added advantage of being relatively easy and is a routine component of patients admitted to ICU. In a recent study on the sepsis-induced hemostatic changes, positive correlation was demonstrated between D-dimer and SOFA score [36]. D-dimer is a fibrin degradation product (FDP) and is a small protein fragment resulting from the breakdown of a fibrin clot (or thrombus). The D-dimer test is now routinely used in the first-line assessment of patients suspected of suffering VTE [37].

One of the primary limitations of our study is its retrospective nature and limited sample size. A larger sample size could have validated better the various risk factors for the development of VTE. In addition, our study was conducted in a single centre and therefore the collected data are subject confounding that may influence our outcomes. A multicentre study with a larger sample size may be necessary to eliminate deviations in the results and to confirm the current findings.

\section{Conclusion}

Our findings suggest that in patients admitted to the ICU, CRE infection is a strong trigger to the development of VTE, and draw the attention of the treating clinicians to prioritise these infections in the management protocols to control infection-driven VTE in ICU patients.

\section{Declarations}

\section{Ethical approval and consent to participation:}

The study was approved by the Institutional Review Board, King Abdullah International Medical Research Centre, King Saud bin Abdulaziz University for Health Sciences, National Guard Health Affairs and informed consent was waived. All procedures performed in the study involving human participants were in accordance with the ethical standards of the institutional and/or national research committee and with the 1964 Helsinki declaration and its later amendments or comparable ethical standards.

\section{Consent for publication:}

Not applicable

\section{Availability of supporting data:}

The data used for this research are available from the corresponding author on reasonable request and subject to Institutional Review Board guidelines.

\section{Competing interest:}

The authors declare that they have no competing of interests.

\section{Funding:}


No funding

\section{Authors' contribution:}

All authors made substantial contributions to conception and design, acquisition of data, or analysis and interpretation of data; took part in drafting the article or revising it critically for important intellectual content; agreed to submit to the current journal; gave final approval of the version to be published; and agree to be accountable for all aspects of the work.

\section{Acknowledgements:}

We would like to acknowledge the help of the Health Information Management, KAMC.

\section{References}

1. Behravesh S, Hoang P, Nanda A et al. Pathogenesis of thromboembolism and Endovascular Management - Review Article. Thrombosis. 2017, Article ID 3039713, 13 pages

2. Kumar, DR, Hanlin E, Glurich I, et al. Virchow's Contribution to the Understanding of Thrombosis and Cellular Biology. Clin Med Res. 2010; 8: 168-172

3. Roberts LN, Patel RK, Arya R. Venous thromboembolism and ethnicity. Brit J Haematol. 2009; 146: 369-383.

4. Patel RK, Arya R. Venous thromboembolism: racial and ethnic influences. A Review. Therapy. 2008, 5; 169-175.

5. Blondon M, Harrington LB, Righini M. et al. Racial and ethnic differences in the risk of postpartum venous thromboembolism: a population-based, case-control study. J Hemostas Thromb. 2014; 12 : 2002-2007.

6. Zhang C, Zhang Z, Jie Mi 1, Wang X, et al. The Cumulative Venous Thromboembolism Incidence and Risk Factors in Intensive Care Patients Receiving the Guideline-Recommended Thromboprophylaxis. Medicine (Baltimore). 2019 Jun;98(23):e15833. doi: 10.1097/MD.0000000000015833.

7. Geerts WH, Bergqvist D, Pineo GF, et al. Prevention of venous thromboembolism: American College of Chest Physicians Evidence-Based Clinical Practice Guidelines (8th Edition). Chest 2008;1336 Suppl:381s-453s. doi: 10.1378/chest.08-0656.

8. Minet C, Potton L, Bonadona A, et al. Venous thromboembolism in the ICU: main characteristics, diagnosis and thromboprophylaxis. Crit Care 2015;19:287-95. DOI:10.1186/s13054-015-1003-9

9. Iba T, Nisio MD, Levy JH, et al. New criteria for sepsis-induced coagulopathy (SIC) following the revised sepsis definition: a retrospective analysis of a nationwide survey. BMJ Open. 2017;7:e017046. DOI: 10.1136/bmjopen-2017-017046

10. Smeeth L, Cook C, Thomas S, Hall AJ, Hubbard R, Vallance P. Risk of deep vein thrombosis and pulmonary embolism after acute infection in a community setting. Lancet. 2006;367:1075-9 
11. Grainge MJ, West J, Card TR. Venous thromboembolism during active disease and remission in inflammatory bowel disease: a cohort study. Lancet. 2010;375:657-63.

12. Cervantes J, Rojas G. Virchow's Legacy: deep vein thrombosis and pulmonary embolism. World J Surg. 2005;29(Suppl. 1):S30-4.

13. Bhagat K, Moss R, Collier J, Vallance P. Endothelial "stunning" following a brief exposure to endotoxin: a mechanism to link infection and infarction? Cardiovasc Res. 1996;32:822-9.

14. Cohen AT, Davidson BL, Gallus AS, et al. Efficacy and safety of fondaparinux for the prevention of venous thromboembolism in older acute medical patients: randomised placebo controlled trial. BMJ 2006;332:325-9. DOI: 10.1136/bmj.38733.466748.7C

15. Leizorovicz A, Cohen AT, Turpie AG, et al. Randomized, placebo-controlled trial of dalteparin for the prevention of venous thromboembolism in acutely ill medical patients. Circulation 2004;110:874-9. DOI: 10.1161/01.CIR.0000138928.83266.24

16. Samama M.M., Cohen A.T., Darmon J.Y., Desjardins L., Eldor A., Janbon C. et al. A comparison of enoxaparin with placebo for the prevention of venous thromboembolism in acutely ill medical patients. Prophylaxis in medical patients with enoxaparin study group. N. Engl. J. Med. 1999; 341: 793-800.

17. Cook D, Meade M, Guyatt G, Walter S, Heels-Ansdell D, Warkentin TE, et al. Dalteparin versus unfractionated heparin in critically ill patients. N Engl J Med. $2011 ; 364: 1305-14$.

18. Patel R, Cook DJ, Meade MO, Griffith LE, Mehta G, Rocker GM, et al. Burden of illness in venous thromboembolism in critical care: a multicenter observational study. J Crit Care. 2005;20:341-7.

19. Knaus WA, Draper EA, Wagner DP, Zimmerman JE. (1985). APACHEIl: a severity of disease classification system. Crit Care Med.;13(10):818-29

20. Vincent J-L, Moreno R, Takala J, et al. (1996). The SOFA (Sepsis-related Organ Failure Assessment) score to describe organ dysfunction/failure. Intensive Care Med.;22(7):707-710

21. Heyland D, Dhaliwal R, Jiang X, Day A. Identifying critically ill patients who benefit the most from nutrition therapy: the development and initial validation of a novel risk assessment tool. Crit Care. 2011;15:R268. doi: 10.1186/cc10546

22. Barzilai-Birenboim S, Arad-Cohen N, Nirel N. et al Thrombophilia screening and thromboprophylaxis may benefit specific ethnic subgroups with paediatric acute lymphoblastic leukaemia. Brit $\mathrm{J}$ Haematol. 2019; 184: 994-998

23. Crowther MA, Cook DJ, Griffith LE, Devereaux PJ, Rabbat CC, Clarke FJ, et al. Deep venous thrombosis: clinically silent in the intensive care unit. J Crit Care. 2005;20:334-40.

24. Cook $D$, Crowther $M$, Meade $M$, Deep venous thrombosis in medical-surgical critically ill patients: prevalence, incidence, and risk factors. Crit Care Med. 2005;33:1565-71.

25. McLeod AG, Geerts W. Venous thromboembolism prophylaxis in critically ill patients. Crit Care Clin. 2011 Oct;27(4):765-80. 
26. Schmidt, M, Horvath-Puho E, Thomsen RW, et al. Acute infections and venous thromboembolism. J Int Med. 2012; 271: 608-618.

27. Grimnes G, Isaksen T, Vladimir Tichelaar YIG, Braekkan SK, Hansen JB. Acute infection as a trigger for incident venous thromboembolism: Results from a population-based case-crossover study. Res Pract Thromb Hemostas. 2018; 2: 85-92.

28. Schultz MJ, Haitsma JJ, Zhang H, Slutsky AS. Pulmonary coagulopathy as a new target in therapeutic studies of acute lung injury or pneumonia-a review. Crit Care Med. 2006;34:871-7.

29. Kaplan D, Charles Casper t, Gregory Elliott C, et al. VTE Incidence and risk factors in patients with severe sepsis and septic shock. Chest 2015; 148: 1224-1230.

30. Shorr AF, Williams MD. Venous thromboembolism in critically ill patients. Observations from a randomized trial in sepsis. Thromb Haemost. 2009 Jan;101(1):139-44.

31. Hong KC, Kim H, Kim JY et al. Risk factors and incidence of deep vein thrombosis in lower extremities among critically ill patients. J Clin Nursing. 2012, 21, 1840-1846.

32. Rogers MA, Levine DA, Blumberg N, Flanders SA, Chopra V, Langa KM. Triggers of hospitalization for venous thromboembolism. Circulation. 2012 May 1; 125(17):2092-9. doi:

10.1161/CIRCULATIONAHA.111.084467.

33. Marshall BE, Hanson CW, Frasch F, Marshall C. Role of hypoxic pulmonary vasoconstriction in pulmonary gas exchange and blood flow distribution. 2. Pathophysiology. Intensive Care Med. 1994; 20: $379-89$.

34. Levi M , van der Poll T, Schultz M. Infection and inflammation as risk factors for thrombosis and atherosclerosis. Semin Thromb Hemost. 2012 ;38(5):506-14.

35. Iba T, Arakawa M, Mochizuki K. Usefulness of Measuring Changes in SOFA Score for the Prediction of 28-Day Mortality in Patients With Sepsis-Associated Disseminated Intravascular Coagulation. Clin Appl Thromb Hemost. 2019; 25: 1-6.

36. Hanna S, Giri,1 N, Aman AK, et al. Hemostasis changes and its relationship with SOFA score in sepsis patients. Bali Med J. 2019; 8: 496-500.

37. Lippi G, Bonfanti L, Saccenti $C$ et al. Causes of elevated D-dimer in patients admitted to a large urban emergency department. Europ J Intern Med. 2014; 25: 45-48.

\section{Figures}




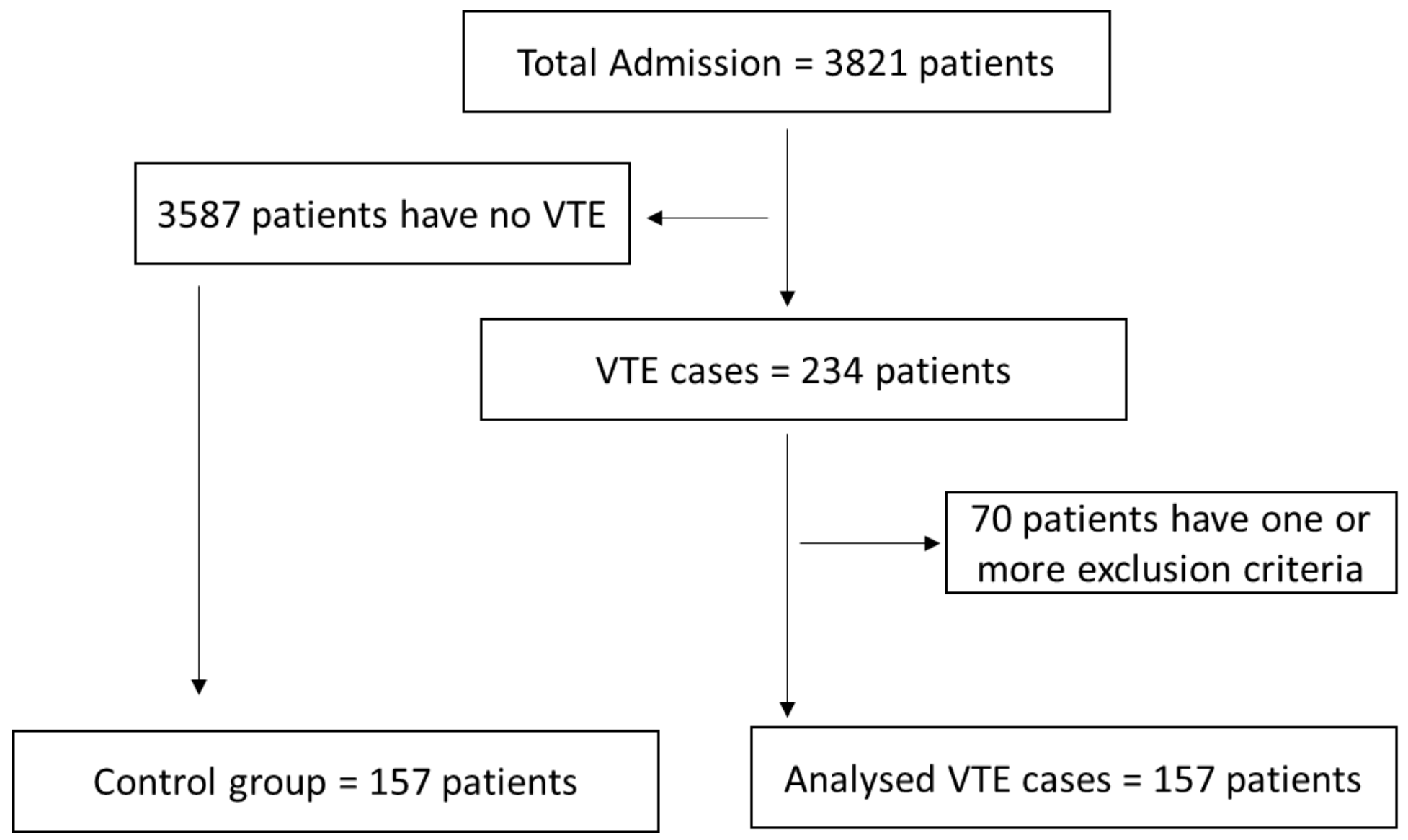

Figure 1

Flow chart of the study population. 